\title{
Sigma Signal for Hybrid Baryon Decay
}

\author{
Leonard S. Kisslinger \\ Department of Physics, Carnegie Mellon University \\ Pittsburgh, PA 15213, USA and \\ Los Alamos National Laboratory, Los Alamos, NM 87545, USA \\ and \\ Zhenping $\mathrm{Li}$
}

Physics Department Peking University, Beijing 100871, P. R. China

July 12, 2021

\begin{abstract}
We develop an ansatze of the sigma enhancement of the $\mathrm{I}=0, \mathrm{~L}=0 \pi-\pi$ scattering amplitude as arising from a low-energy glueball pole. Using this picture we estimate the $\pi^{0} \pi^{0}$ to $\pi^{0}$ branching ratio for the decays of the Roper resonance, which we previously found to be a hybrid in our QCD sum rule calculation.
\end{abstract}

PACS numbers: 12.39.Mk, 11.55.Hx, 14.20.Gk 
In an investigation of scalar glueballs one of us has has found[1] that a pure scalar glueball has a mass 300-600 MeV, while solutions for mixed scalar glueballs and mesons are at a much higher energy, consistent with the $\mathrm{f}_{0}(1500)$ for the mainlyglueball solution and with the $\mathrm{f}_{0}(1370)$ and $\mathrm{a}_{0}(1450)$ for the mainly-meson solution. Recently there has been a great deal of experimental activity in glueball searches [2, 3]. One of the very interesting and we feel most important observations is the enhancement of the $\sigma$ mode in the decays of the glueball candidates, such as the dominance of the $\sigma \sigma$ intermediate states in the decays of the glueball candidates $f_{0}(1500) \rightarrow 4 \pi$ and $f_{0}(1710) \rightarrow 4 \pi$, 4 , 4. The recent analysis by the BES collaboration 4 also suggest that the tensor glueball candidates $\xi(2230)$ also has a large $4 \pi$ branching ratio that is dominated by the $\sigma f_{2}(1270)$ intermediate state.

By the " $\sigma$ " we mean in the present paper the enhancement in the $\pi-\pi \mathrm{I}=0, \mathrm{~L}=0$ amplitude, for which a pole has been found in the analysis of Zou and Bugg[5]. This broad pole occurs near the light scalar glueball found in our work [1], and the work of others using QCD sum rule methods [6]. We propose that the physical origin of the $\sigma$ enhancement is the light scalar gluonic mode, and that this could be a good signal for the study of gluonic structure of hadrons. This also provides an explanation of the absence of light, narrow glueball in lattice gauge calculations, since the strong coupling to the $\pi-\pi$ channel, which results in about a $700 \mathrm{MeV}$ width must be included for a physical solution. Note the the mixed glueball-meson states, predicted to be at higher energies in the region of scalar mesons[国, 7], are quite far from this coupled-channel sigma and would not be involved.

In the present work we use this picture of a scalar glueball mode coupled to the $\sigma$ channel for the decay of hybrid baryons. Recently we carried out a QCD sum rule calculation 8] to find the hybrid baryon with quantum numbers $J^{P}=1 / 2^{+}, I=$ $1 / 2$, the same as the nucleon and the $\mathrm{P}_{11}(1440)$, the "Roper". This consisted as an 
improvement over earlier work using the QCD sum rule methods 9, 10]. Since that work we have considered currents of the form $c_{1} \eta_{N}+c_{2} \eta_{H}$, where $\eta_{N}$ and $\eta_{H}$ are the nucleon and hybrid currents, defined below, and have shown that the nucleon has a very small hybrid component [1], which indicates that using a pure hybrid current for the Roper is reasonable.

Because of convenience in treating renormalization we use a different current operator for the $J^{P}=1 / 2^{+}, I=1 / 2$ hybrid state than in Ref [8]. It is

$$
\eta_{H}(x)=\epsilon^{a b c}\left(u^{a}(x) C \gamma^{\mu} u^{b}(x)\right) i \sigma^{\alpha \beta} \gamma^{\mu} \gamma^{5} G_{\alpha \beta}^{d}(x)\left(T^{d} d(x)\right)^{c}
$$

where $u(x)$ and $d(x)$ are operators of the $u$ and $d$ quarks, $G_{\mu \sigma}^{d}(x)$ is a gluon field strength, $a, b, c=1,2,3$ are the color indices, $C$ is the charge conjugation matrix, and $T^{d}=\lambda^{d} / 2$ is the generator of the $S U(3)$ color group. For the calculation of the pion and sigma decays we use the external field two-point method 12], which has been used in many calculations of hadronic coupling constants, including the strong and weak pion-nucleon coupling constant 13]. Using a two-point function in an external $\mathrm{J}_{\Gamma}$ field to represent the coupling of the current operator to the nucleon and the Roper, with the current of Eq. 1 lead to two independent invariant functions;

$$
\begin{aligned}
\Pi^{s t}(q) & =i \int e^{i q x}\left\langle 0\left|T\left(\eta_{H}^{s}(x) \bar{\eta}_{N}^{t}(0)\right)_{\Gamma}\right| 0\right\rangle \\
& =\Pi_{1}^{\Gamma}\left(q^{2}\right) \hat{q}^{s t}+\Pi_{2}^{\Gamma}\left(q^{2}\right) \delta^{s t}
\end{aligned}
$$

where s,t are Dirac indices, $\hat{q}^{s t}=\left(\gamma_{\mu}\right)^{s t} q^{\mu}$, and $\eta_{N}$ is the nucleon current. The details of the solution for the mass of the hybrid are given in Ref [8].

The coefficients of the operator product expansion (O.P.E.) are calculated to dimension 6 . In addition to the leading perturbative contributions we include the two quark condensate terms, which are the largest nonperturbative terms. For the pion decay the current $\mathrm{J}_{\pi}=\mathrm{i} \bar{q} \gamma_{5} q$ and the $\Pi_{2}$ correlator are used. After the Borel transform 
we find the following sum rule

$$
\begin{aligned}
\Pi_{2}^{\pi}\left(M^{2}\right) & =G_{\pi} \frac{\lambda_{N} \lambda_{H}}{\left(M^{N}+M^{H}\right)}\left(M_{H} e^{-M_{H}^{2} / M^{2}}-M_{N} e^{-M_{N}^{2} / M^{2}}\right)(2 \pi)^{4} \\
& =\frac{11}{12} M^{8} E^{3}-\frac{4 a^{2}}{9} M^{2} E_{0}
\end{aligned}
$$

where $\lambda_{N}, \lambda_{H}$ are the QCD sum rule structure constants, and $\mathrm{G}_{\pi}$ is the pi-Ropernucleon coupling. The susceptibility term is small and has been neglected. For the sigma decay we use the current $\mathrm{J}_{\sigma}$, with the glue-sigma coupling, $\mathrm{g}_{\sigma}$, given by

$$
<G_{\alpha \beta}^{a} J_{\sigma} G_{a}^{\alpha \beta}>=g_{\sigma}<G_{\alpha \beta}^{a} G_{a}^{\alpha \beta}>
$$

The glue-sigma coupling constant can be extracted from the $\pi-\pi$ T-Matrix of Ref [5]. After subtracting the higher resonances they find a fit to the $\pi-\pi$ amplitude of a Breit-Wigner form:

$$
T^{\pi \pi(\sigma)}=\frac{\sqrt{s} \Gamma_{\sigma}(s) / 2}{s-M_{\sigma}^{2}+i \sqrt{s} \Gamma_{\sigma}(s) / 2}+T(\text { background }) .
$$

The analysis of Ref [5] finds the sigma pole at 370 - 356i. Using our ansatze we determine the sigma- gluonic coupling constant, $\mathrm{g}_{\sigma}$ from the width of this pole, giving

$$
g_{\sigma} \simeq 700 \mathrm{MeV}
$$

We obtain for the $\Pi_{2}$ correlator

$$
\begin{aligned}
\Pi_{2}\left(M^{2}\right) & =G_{\sigma} \frac{\lambda_{N} \lambda_{H}}{\left(M^{H}-M^{N}\right)}\left(e^{-M_{N}^{2} / M^{2}}-e^{-M_{H}^{2} / M^{2}}\right)(2 \pi)^{4} \\
& =g_{\sigma}\left(\frac{7}{30} M^{8} E^{3}-\frac{a^{2}}{3} M^{2} E_{0}\right)
\end{aligned}
$$

In Eq.(7) the parameter $\mathrm{G}_{\sigma}$ is the $\mathrm{H}-\mathrm{N}-\sigma$ coupling constant which apears in the numerator of the double-pole term in the phenomenological dispersion relationship.

From $\mathrm{G}_{\sigma}$ and $\mathrm{G}_{\pi}$ the $\pi$ to $\sigma$ branching ratio can be determined. Using the standard values of the parameters, we find for the $\sigma / \pi$ branching ratio

$$
\frac{\Gamma_{\sigma}}{\Gamma_{\pi}} \simeq 10 \% \cdot R_{p}
$$


where $R_{p}$ is the ratio of phase space factors. This is consistent with the present experimental limits [14. Indeed, the data suggest that the width of the Roper resonance $P_{11}(1440)$ decaying into $\sigma N$ final states is generally an order of magnitude larger than those of other resonances. This could be another signature of a hybrid Roper resonance in addition to its electromagnetic transition properties 15]. Thus, further experimental investigations of $P_{11}(1440) \rightarrow \pi^{0} \pi^{0} N$ [to select the $\mathrm{I}=0$ channel

and eliminate $\Delta$ and $\rho$ backgrounds] over an energy range to map out the $\sigma$ would be a very important channel to study the structure of the Roper resonance. In general, we suggest that the measurement of $\sigma$ decays of baryons and mesons is a signal for the gluonic content of hadrons.

We would like to thank Mikkel Johnson for helpful discussions. This work was supported by National Science Foundation grants PHY-9319641 and INT-9514190, and by the Department of Energy.

\section{References}

[1] L.S. Kisslinger, J. Gardner and C. Vanderstraeten," Mixed Scalar Glueballs and Mesons", to be published in Phys. Lett. B (1997).

[2] Crystal Barrel Collaboration, Phys. Lett. B 355, 425 (1995).

[3] D. V. Bugg, et al, Phys. Lett. 353B, 378 (1995).

[4] Zhu Yucan, BES J/ $\Psi$ group, to appear on The Proceedings of the 6th Conference On The Intersection of Particle and Nuclear Physics, Big Sky, Montana (1997).

[5] B.S. Zou and D.V.Bugg, Phys. Rev. D 50, 591 (1994). 
[6] C.A. Dominguez and N. Paver, Zeit. Phys. C 31, 591 (1986); J. Bordes, V. Gimenez and J.A. Penarrocha, Phys. LettB 223, 251 (1989); J. Liu and D. Liu, J. Phys. G:Nucl. Part. Phys. 19, 373 (1993).

[7] S. Narison, hep-ph/9612457.

[8] L.S. Kisslinger and Z. Li, Phys. Rev. D 51, R5986 (1995).

[9] A.P. Martynenko, Sov. J. Nucl. Phys. 54, 488 (1991).

[10] V.M. Braun, P. Gornicki, L. Mankiewicz and A. Schafer, Phys. Lett.B 302, 291 (1993).

[11] L.S. Kisslinger, "Gluonic Hadrons", to be published in the Proceedings of the International Conference on Quark Lepton Nuclear Physics, Osaka, Japan (1997).

[12] B.L. Ioffe and A.V. Smilga, Nucl. Phys. B 232, 109 (1984).

[13] E.M. Henley, W-Y. P. Hwang and L.S. Kisslinger, Phys. Lett. B 367, 21 (1996).

[14] Review of Particle Properties, Phys. Rev. D 45 (1992).

[15] Zhenping Li, V. Burkert and Zhujun Li, Phys. Rev. D46, 70 (1992); Zhenping Li, Phys. Rev. D44, 2841 (1991). 